\title{
Mielopatía Espondilótica Cervical Como un Imitador de Accidente Cerebrovascular
}

\author{
Cervical Spondylotic Myelopathy As A Stroke Mimic
}

Gustavo Gaitan-Quintero, Mario Montoya-Jaramillo²

\section{Resumen}

La mielopatía espondilótica cervical (MEC) es la afección de la médula espinal no traumática más frecuente en mayores de 55 años. Este trastorno es debido a la compresión de la médula espinal por un canal cervical estenótico. Se describe un paciente hipertenso de 55 años con un cuadro clínico que simuló un accidente cerebrovascular, debido a una presentación súbita de un déficit motor braquiocrural derecho, con elevación de cifras tensionales en rango de crisis hipertensiva. Horas después presentó igual déficit motor contralateral. La tomografía computarizada y resonancia magnética cerebral no mostraron anormalidad. Fue llamativo el hallazgo de atrofia en los músculos interóseos. Se realizó resonancia magnética cervical que evidenció un canal estrecho, con hiperintensidad medular en secuencias T2 de niveles C2 a C6. Las causas más frecuentes de imitadores de ictus son los tumores y los trastornos tóxico metabólicos. Los trastornos medulares representan $1.7 \%$ de todos los casos, siendo la MEC una causa rara.

Palabras clave: Mielopatía espondilótica cervical, Imitador de ictus, Mielitis.

\section{Abstract}

Cervical spondylotic myelopathy (CSM) is the most common nontraumatic spinal cord condition in people over 55 years of age. This disorder is due to compression of the spinal cord by a stenotic cervical canal. A 55-year-old hypertensive patient with a clinical picture that simulated a stroke is described, due to a sudden onset of a right brachiocrural motor deficit, with elevated blood pressure levels in the range of hypertensive crisis. Hours later the patient presented the same motor deficit contralaterally: Computed tomography and magnetic resonance imaging of the brain showed no abnormality. The finding of atrophy in the interosseous muscles was striking. Cervical magnetic resonance imaging was performed showing a narrow spinal canal, with spinal cord hyperintensity in T2 sequences at levels $\mathrm{C} 2$ to $\mathrm{C} 6$. The most frequent causes of stroke mimics are tumors and metabolic toxic disorders. Spinal disorders represent $1.7 \%$ of all cases, CSM being a rare cause.

Keywords: Cervical spondylotic myelopathy, Stroke mimic, Spinal cord injury.

Rev. Ecuat. Neurol. Vol. 29, N², 2020

\section{Introducción}

La mielopatía espondilótica cervical (MEC) es un trastorno de la médula espinal secundario a cambios degenerativos de la columna cervical, que ocurre por una compresión medular secundaria a un canal cervical estenótico que genera cambios isquémicos crónicos. Es la causa más frecuente de disfunción y deterioro de la médula espinal no traumática en adultos mayores de 55 años. Se estima una incidencia y prevalencia de 4.1 y 60.5 por 100,000 habitantes respectivamente. Usualmente tiene una presentación clínica progresiva, ocasionalmente se ha descrito una progresión rápida. A continuación se describe una inusual presentación súbita que simuló un ictus.

\section{Caso clínico}

Hombre de 55 años, operador de obras, sin antecedentes médicos de importancia; mientras se encontraba en su domicilio, en bipedestación, sin previamente realizar
'Servicio de Neurología. Clínica Cartagena del Mar. Universidad del Sinú. Cartagena Colombia.

${ }^{2}$ Departamento de Medicina Interna. Clínica Cartagena del Mar. Universidad del Sinú. Cartagena Colombia.
Correspondencia:

Gustavo Adolfo Gaitán Quintero. Servicio de Neurología, Clínica Cartagena del Mar.

Universidad del Sinú Cartagena. Transversal 54 \#41-117

Cartagena De Indias, Colombia. gustavoagaitanq@hotmail.com 
algún movimiento cervical, sin traumatismo previo presenta súbita aparición de déficit motor braquial izquierdo que en minutos progresa a braquiocrural, asociado a dolor craneofacial intenso y pulsátil, de predominio occipital irradiado a región cervical posterior. El paciente decide ir a urgencias e ingresa por sus propios medios debido a la persistencia de los síntomas después de automedicarse paracetamol. Al ingreso se documenta una paresia braquiocrural izquierda $3 / 5$, sin otros hallazgos adicionales al examen neurológico. Presentaba glucometria normal, cifra tensional de 220/100 mmh. La tomografía cerebral fue normal. No se realizó trombolisis por estar fuera de la ventana terapéutica e ingresa a terapia intensiva con diagnóstico de ictus isquémico asociado a una crisis hipertensiva. Durante la hospitalización -a las 48 horas del inicio de los síntomas- se añade la aparición de paresia braquiocrural derecha 4/5. La resonancia magnética cerebral contrastada con secuencia de difusión era normal; otros exámenes de función hepática, función renal, electrolitos, electrocardiograma holter, doppler de vasos de cuello y ecocardiograma fueron normales.

Era llamativo la presencia atrofia en los músculos interóseos de las manos y debido a las características del déficit motor se realizó resonancia cervical simple donde se evidencio hallazgos típicos de una MEC con estrechez severa del canal cervical, compresión medular y hiperintensidad medular en T2 (fig. 1 y fig. 2). Durante los siguientes días de hospitalización el paciente presentó mejoría del dolor craneofacial con la administración de naproxeno. Posteriormente, se intervino quirúrgicamente resultando en mejoría clínica y resolución casi completa de los síntomas. Persistió una monoparesia crural derecha $4 / 5$ y pudo volver a sus actividades cotidianas.

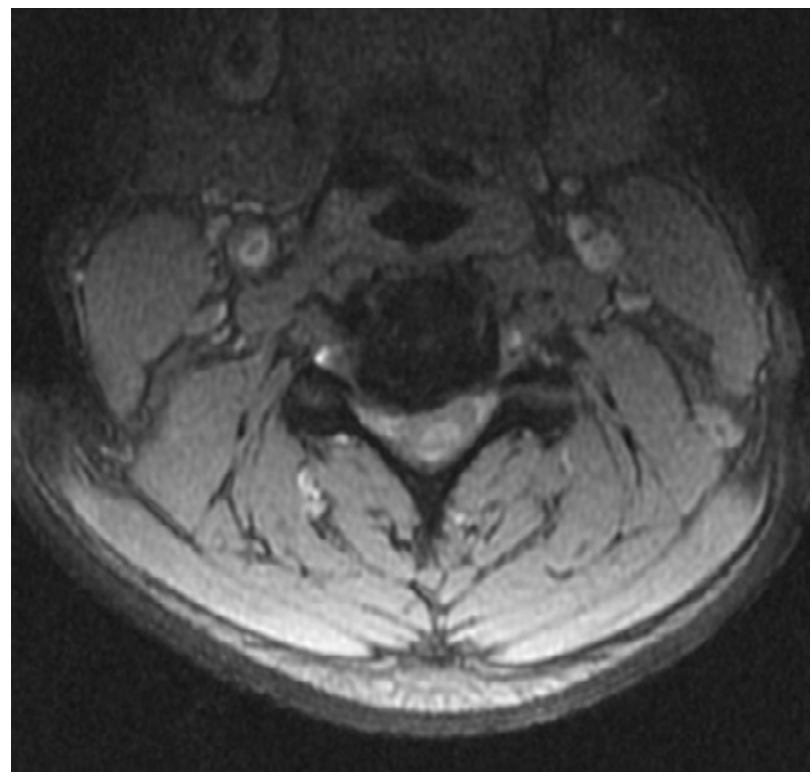

Figura 1. Corte axial de resonancia magnética cervical en el que se aprecia severa estrechez del canal.

\section{Discusión}

El término accidente cerebrovascular o ictus es usado para la aparición súbita de síndromes y signos neurológicos focales que persisten durante más de 24 horas debido a trastornos cerebrovasculares. Existen trastornos que pueden imitar los signos clínicos de un ictus. Su diagnóstico y manejo inadecuado pueden derivar en las mismas complicaciones de una isquemia cerebral. Los frecuentemente descritos incluyen trastornos tóxicos o metabólicos como hipoglucemia, hipercalcemia, hiponatremia, uremia, encefalopatía hepática, hipertiroidismo; tumores cerebrales (gliomas, meningiomas y adenomas entre los más comunes), trastornos infecciosos como meningoencefalitis, desórdenes desmielinizantes, migraña, convulsiones y trastornos psicógenos. ${ }^{3}$ Estos trastornos imitadores pueden estar en un 30\% de los pacientes con un diagnóstico inicial de ictus, ${ }^{4}$ donde los trastornos neoplásicos y tóxico metabólicos se han descrito entre las causas más frecuentes. ${ }^{3}$ Los síntomas más frecuentes descritos en estos trastornos son afasia, vértigo, síncope, diplopía, disartria, hipo o alteraciones en la deglución..$^{3,5}$ Un estudio de Irán con 1985 pacientes con diagnóstico inicial de ictus encontró que 295 pacientes (15\%) presentaban trastornos imitadores, siendo más frecuentes en el rango de 61 a 80 años de edad, donde los trastornos de la médula espinal representaron un $1.7 \%$.

La MEC es una causa frecuente de deterioro de la médula espinal en la población de edad avanzada. Normalmente es un diagnóstico diferencial de otras causas de mielopatía como la Esclerosis Lateral Amiotrófica, deficiencia de vitamina B12, Paraparesia Espástica Tropical, Esclerosis Múltiple, entre otras. La causa de este trastorno es debido a la compresión crónica de la médula

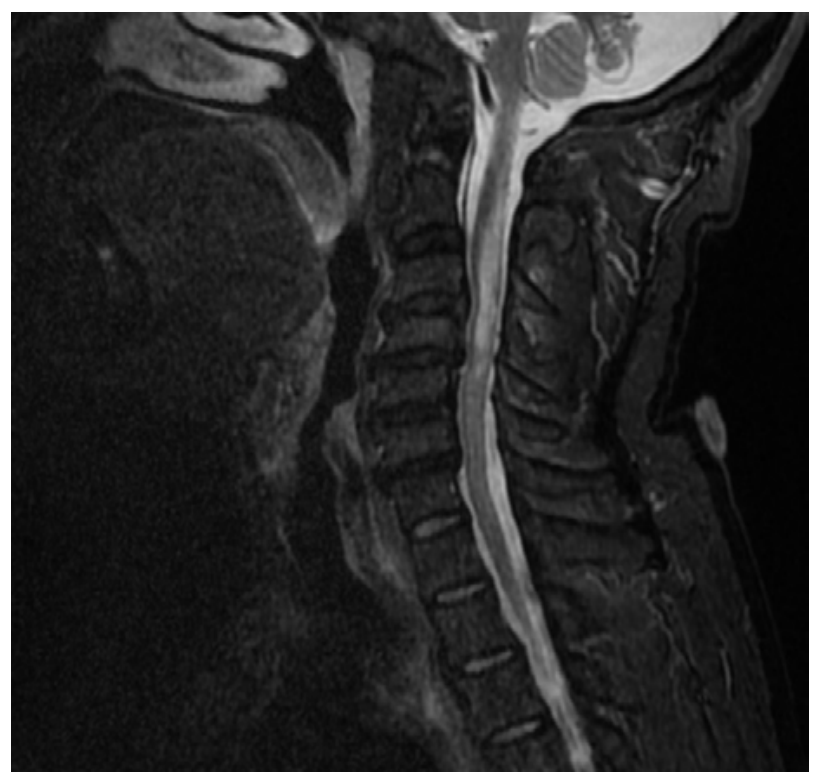

Figura 2. Corte sagital de resonancia magnética cervical que muestra hiperintensidad medular en $\mathrm{T} 2$. 
espinal por un canal cervical estenótico. Esto ocurre por cambios degenerativos en la columna cervical en un proceso normal asociado con el envejecimiento. La compresión y los cambios isquémicos locales del parénquima medular debido a alteraciones en la vasculatura arterial y venosa provocarían la mielopatía. Entre los cambios isquémicos locales se observa inducción de apoptosis de los oligodendrocitos con desmielinización, afectándose principalmente los tractos corticoespinales y el cordón medular posterior, además de alteración de la barrera hematoespinal? La presentación clínica es insidiosa, con un curso clínico escalonado y crónico en la mayoría de los pacientes; sin embargo, se ha descrito una progresión rápida en el $5 \%$ de los pacientes. ${ }^{8}$ Entre las manifestaciones clínicas más frecuentes están la pérdida del control motor fino de los miembros superiores, inestabilidad de la marcha, debilidad y el dolor de cuello con un rango de movimiento reducido, pérdida de control del esfínter urinario; también son frecuentes la espasticidad de los miembros inferiores, alteración de la propiocepción. Es característica la atrofia de músculos interóseos y tenares con la abolición de los reflejos tendinosos, cuando la compresión ocurre por debajo del nivel C5. Por el contrario, si la compresión ocurre por encima de $\mathrm{C} 5$, pueden aparecer hiperreflexia y espasticidad de los miembros superiores, signo de Hoffman, reflejo estilorradial invertido; además puede estar presente el signo de Lhermitte.,7,9

El diagnóstico requiere de una alta sospecha clínica, siendo la resonancia magnética es el examen de elección. Los hallazgos típicos son un canal espinal cervical estenótico con compresión medular e hiperintensidad difusa del parénquima en secuencia T2; ocasionalmente se puede observar hipointensidad del parénquima en T1, lo que se asocia a cambios degenerativos irreversibles, necrosis y mielomalacia. Además de ser útil la resonancia magnética para valorar los cambios degenerativos de la columna vertebral, también es indispensable en la toma de decisiones en los abordajes quirúrgicos. ${ }^{10,11} \mathrm{El}$ tratamiento quirúrgico es el más recomendado y efectivo. Es mandatorio en los casos moderados a severos, comprende múltiples abordajes y procedimientos quirúrgicos. El manejo no quirúrgico dispone de analgésicos, antinflamatorios, inmovilización cervical, fisioterapia. ${ }^{12}$

\section{Conclusiones}

La MEC es una causa frecuente de deterioro de la médula espinal en la población de edad avanzada. Tiene múltiples formas de presentación y manifestaciones clínicas. Además de ser un diagnóstico diferencial de otras causas de mielopatía, también puede tener una presentación inusual, imitando un ictus como lo demuestra este caso.

\section{Referencias}

1. Vilaça, C., Orsini, M., Araujo Leite, M., de Freitas, M., Davidovich, E., Fiorelli, R., Fiorelli, S., Fiorelli, C., Oliveira, A., \& Pessoa, B. (2016). Cervical spondylotic myelopathy: what the neurologist should know. Neurology International, 8(4).

2. Nouri A, Tetreault L, Singh A, Karadimas SK, Fehlings MG. Degenerative Cervical Myelopathy: Epidemiology, Genetics, and Pathogenesis. Spine (Phila Pa 1976). 2015;40(12):E675-E693.

3. Hatzitolios A, Savopoulos C, Ntaios G, Papadidaskalou F, Dimitrakoudi E, Kosmidou M, et al. Stroke and conditions that mimic it: a protocol secures a safe early recognition. Hippokratia 2008;12:98-102.

4. Merino JG, Luby M, Benson RT, et al. Predictors of acute stroke mimics in 8187 patients referred to a stroke service. J Stroke Cerebrovasc Dis. 2013;22:e397-e403.

5. A. Kose, T. Inal, E. Armagan, A.B. KiyakR. Demir, Conditions that mimic stroke in elderly patients admitted to the emergency department, J. Stroke Cerebrovasc. Dis. 22 (2013) e522-e527

6. Hosseininezhad M, Sohrabnejad R. Stroke mimics in patients with clinical signs of stroke. Caspian J Intern Med. 2017; 8(3): 213-216.

7. Gibson J, Nouri A, Krueger B, Lakomkin N, Nasser R, Gimbel D, Cheng J. Degenerative cervical myelopathy: a clinical review. Yale J Biol Med. 2018;91:43-8.

8. St. Clair S, Bell GR. Natural history of cervical spondylotic myelopathy. Semin Spine Surg 2007;19:2-5.

9. Lavelle WF, Bell GR. Mielopatía cervical: historia y exploración física. Semin Spine Surg 2007; 19: 6-11.

10. Tetreault LA, Dettori JR, Wilson JR, et al. Systematic review of magnetic resonance imaging characteristics that affect treatment decision making and predict clinical outcome in patients with cervical spondylotic myelopathy. Spine (Phila Pa 1976) 2013; 38 (22 suppl 1):S89-S110.

11. Machino M, Imagama S, Ando $\mathrm{K}$, Kobayashi $\mathrm{K}$, Ito $\mathrm{K}$, Tsushima $\mathrm{M}$, Matsumoto $\mathrm{A}$, Morozumi $\mathrm{M}$, Tanaka S, Ito K, Kato F, Nishida Y, Ishiguro N (2018) Image diagnostic classification of magnetic resonance T2 increased signal intensity in cervical spondylotic myelopathy: clinical evaluation using quantitative and objective assessment. Spine 43:420-426.

12. Wilson JR, Tetreault LA, Kim J, et al. State of the art in degenerative cervical myelopathy: an update on current clinical evidence. Neurosurgery. 2017;80(3S):S33- 45. 